\title{
Stability of Fuzzy Dynamical Systems via Lyapunov Functions
}

\author{
Abdelati El Allaoui (D), Said Melliani (D), and Lalla Saadia Chadli \\ Sultan Moulay Slimane University, Department of Mathematics, Laboratory of Applied Mathematics \& Scientific Calculus, \\ Beni Mellal, P.O. Box 523, Morocco \\ Correspondence should be addressed to Abdelati El Allaoui; elallaoui199@gmail.com
}

Received 29 July 2019; Revised 24 February 2020; Accepted 4 July 2020; Published 1 August 2020

Academic Editor: Patricia J. Y. Wong

Copyright (c) 2020 Abdelati El Allaoui et al. This is an open access article distributed under the Creative Commons Attribution License, which permits unrestricted use, distribution, and reproduction in any medium, provided the original work is properly cited.

\begin{abstract}
The purpose of this paper is to introduce the concept of fuzzy Lyapunov functions to study the notion of stability of equilibrium points for fuzzy dynamical systems associated with fuzzy initial value problems, through the principle of Zadeh. Our contribution consists in a qualitative characterization of stability by a study of the trajectories of fuzzy dynamical systems, using auxiliary functions, and they will be called fuzzy Lyapunov functions. And, among the main results that have been proven is that the existence of fuzzy Lyapunov functions is a necessary and sufficient condition for stability. Some examples are given to illustrate the obtained results.
\end{abstract}

\section{Introduction}

The topics of fuzzy dynamical systems have been rapidly growing in recent years, and the first characterization of this concept is presented in [1]. Fuzzy dynamical systems have been dealt with different approaches. Some authors use the extension principle in order to extend deterministic systems of differential equations to the fuzzy case [2-7]. Others construct the fuzzy dynamical systems by using a family of differential inclusions [8-10].

The notion of stability for this type of dynamical systems has been studied by many researchers $[3,7,8,10-15]$. In [12], the authors introduced the concept of fuzzy equilibrium point stability of fuzzy initial value problems defined $\mathscr{F}\left(\mathbb{R}^{n}\right)$, where $\mathscr{F}\left(\mathbb{R}^{n}\right)$ is the fuzzy set space on $\mathbb{R}^{n}$, by using equilibrium points. The authors in [10] have studied the stability of invariant sets for dynamical systems. According to them, equilibrium points have been considered as a special case of fuzzy invariant sets. These approaches have some shortcomings because they require knowledge of the explicit form of the solution of the fuzzy differential equation, which is not always possible to find.

The aim of this paper is to present an alternative approach to these methods to prove the stability of an equilibrium point by introducing fuzzy Lyapunov functions, which are defined on $\mathscr{F}\left(\mathbb{R}^{n}\right)$ and obtained by the Zadeh's extension of a Lyapunov function on $\mathbb{R}^{n}$. Moreover, without having the explicit solution of the fuzzy problem. This is an important point because the fuzzy space $\mathscr{F}\left(\mathbb{R}^{n}\right)$ is bigger than the space $\mathbb{R}^{n}$. Thus, the case of Lyapunov functions on $\mathbb{R}^{n}$ will be particular cases of fuzzy Lyapunov functions because $\mathbb{R}^{n}$ is a classic subset of $\mathscr{F}\left(\mathbb{R}^{n}\right)$.

\section{Preliminaries}

In this section, we recall some basic tools of fuzzy set theory.

Let $\mathrm{P}_{K}\left(\mathbb{R}^{n}\right)$ denote the family of all nonempty compact convex subsets of $\mathbb{R}^{n}$.

The distance between two nonempty bounded subsets $A$ and $B$ of $\mathbb{R}^{n}$ is defined by the Hausdorff metric:

$$
d(A, B)=\max \{\rho(A, B), \rho(B, A)\},
$$

where $\rho(A, B)=\sup _{a \in A} \inf _{b \in B}\|a-b\|$ and $\|$.$\| denotes the$ usual Euclidean norm in $\mathbb{R}^{n}$.

$\left(\mathrm{P}_{K}\left(\mathbb{R}^{n}\right), d\right)$ is a complete and separable metric space (see [16]).

Remember that a fuzzy subset $u$ of a classical set $X$ is characterized by a mapping $\mu_{u}: X \longrightarrow[0,1]$ called the membership function of $u$, and $\mu_{u}(x)$ means the degree of membership of $x$ in $u$. 
In the following, to simplify, we denote by $u$ the membership function $\mu_{u}$.

The $\alpha$-cuts of a fuzzy set $u$ are defined by

$$
[u]^{\alpha}=\{x \in X: u(x) \geq \alpha\}, \quad \text { for } 0<\alpha \leq 1,
$$

and the support of $u$ is defined by

$$
\operatorname{Supp}(u)=\overline{\{x \in X: u(x)>0\}}=[u]^{0} .
$$

Denote by $\mathscr{F}(X)$ the set of fuzzy subsets of $X$ with nonempty and compact $\alpha$-cuts. We are only interested here in $\mathscr{F}\left(\mathbb{R}^{n}\right)$, so the metric is given by

$$
D(u, v)=\sup _{0 \leq \alpha \leq 1} d\left([u]^{\alpha},[v]^{\alpha}\right) .
$$

Now, we recall some properties of the extension principle.

Definition 1 (Zadeh's extension principle, see $[2,5,6])$. Let $f: X \longrightarrow Z$ be a function, and let $A$ be a fuzzy subset of $X$. Zadeh's extension of $f$ is the function $\hat{f}: \mathscr{F}(X) \longrightarrow \mathscr{F}(Z)$ which applied to $A$ gives us the fuzzy subset $\hat{f}(A)$ of $Z$ with the membership

$$
\mu_{\widehat{f}(A)}(z)= \begin{cases}\sup _{x \in f^{-1}(z)} \mu_{A}(x), & \text { if } f^{-1}(z) \neq \varnothing, \\ 0, & \text { if } f^{-1}(z)=\varnothing,\end{cases}
$$

where $f^{-1}(z)=\{x: f(x)=z\}$.

The following result is very useful for fuzzy differential equations (see [17-21]).

Theorem 1 (see $[5,6])$. Let $f: X \longrightarrow Z$ be a continuous function, and let $A$ be a fuzzy subset of $X$. Then, for all $\alpha \in[0,1]$,

$$
[\widehat{f}(A)]^{\alpha}=f\left([A]^{\alpha}\right) .
$$

The idea of the Zadeh's extension approach is as follows. We consider the following fuzzy initial value problem:

$$
\left\{\begin{array}{l}
\frac{\mathrm{d} \mathbf{x}}{\mathrm{d} t}=F(x(t)), \\
x(0)=x_{0} \in \mathscr{F}\left(R^{n}\right),
\end{array}\right.
$$

where $F: \mathscr{F}\left(\mathbb{R}^{n}\right) \longrightarrow \mathscr{F}\left(\mathbb{R}^{n}\right)$ is the Zadeh's extension of a continuous function $f: \mathbb{R}^{n} \longrightarrow \mathbb{R}^{n}$.

A solution of (7) is defined as Zadeh's extension of the deterministic solution $\varphi_{t}\left(x_{0}\right)$ of the initial value problem associated:

$$
\left\{\begin{array}{l}
\frac{\mathrm{d} x}{\mathrm{~d} t}=f(x(t)), \\
x(0)=x_{0} \in \mathbb{R}^{n} .
\end{array}\right.
$$

The fuzzy solution of equation (7) is denoted by $\widehat{\varphi}_{t}\left(\mathbf{x}_{0}\right)$.

The family $\hat{\varphi}_{t}$ satisfies the properties of a flow, and the result is given in the following theorem.
Theorem 2 (see [11]). The fuzzy solution $\widehat{\varphi}_{t}\left(\mathbf{x}_{0}\right)$ verifies the properties:

(1) $\widehat{\varphi}_{0}\left(\mathbf{x}_{0}\right)=\mathbf{x}_{0}$

(2) $\widehat{\varphi}_{t+s}\left(\mathbf{x}_{0}\right)=\widehat{\varphi}_{t}\left(\widehat{\varphi}_{s}\left(\mathbf{x}_{0}\right)\right)$, for all $\mathbf{x}_{0} \in \mathscr{F}\left(\mathbb{R}^{n}\right)$ and $t \in \mathbb{R}_{+}$

So, the family $\widehat{\varphi}_{t}$ defines a flow $\widehat{\varphi}_{t}: \mathscr{F}\left(\mathbb{R}^{n}\right) \longrightarrow \mathscr{F}\left(\mathbb{R}^{n}\right)$, which associates each $\mathbf{x}_{0}$ with a point $\hat{\varphi}_{t}\left(\mathbf{x}_{0}\right)$.

The phase space of $\hat{\varphi}_{t}$ is the metric space $\left(\mathscr{F}\left(\mathbb{R}^{n}\right), D\right)$

$\varphi_{t}$ is continuous with respect to the initial condition, so $\widehat{\varphi}_{t}$ is also continuous

Thus, the family $\widehat{\varphi}_{t}: \mathscr{F}\left(\mathbb{R}^{n}\right) \longrightarrow \mathscr{F}\left(\mathbb{R}^{n}\right)$ is a dynamical system in $\mathscr{F}\left(\mathbb{R}^{n}\right)$, for that it is called a fuzzy dynamical system.

Example 1. Consider the following nonlinear differential equation:

$$
\left\{\begin{array}{l}
x^{\prime}=g(x) \\
x(0)=x_{0} \in \mathbb{R}^{+}
\end{array}\right.
$$

where $g(x)=\delta x(1-x)$.

This system determines the flow $\varphi_{t}: \mathbb{R} \longrightarrow \mathbb{R}$ given by

$$
\varphi_{t}\left(x_{0}\right)=\frac{x_{0}}{x_{0}-\left(x_{0}-1\right) e^{-\delta t}}, \quad \text { for } t \geq 0
$$

We consider the fuzzy initial value problem:

$$
\left\{\begin{array}{l}
\mathbf{x}^{\prime}=\widehat{g}(\mathbf{x}), \\
\mathbf{x}(0)=\mathbf{x}_{0} \in \mathscr{F}\left(\mathbb{R}^{+}\right),
\end{array}\right.
$$

where $\hat{g}$ is the Zadeh's extension of $g$ defined by

$$
[\widehat{g}(\mathbf{x})]^{\alpha}=g\left([\mathbf{x}]^{\alpha}\right)=\left[\delta x_{\alpha}^{-}\left(1-x_{\alpha}^{+}\right), \delta x_{\alpha}^{+}\left(1-x_{\alpha}^{-}\right)\right],
$$

for $[\mathbf{x}]^{\alpha}=\left[x_{\alpha}^{-}, x_{\alpha}^{+}\right]$and $\alpha \in[0,1]$.

For $\mathbf{x}_{0} \in \mathscr{F}(\mathbb{R}), \alpha \in[0,1]$ and $\left[\mathbf{x}_{0}\right]^{\alpha}=\left[x_{0 \alpha}^{-}, x_{0 \alpha}^{+}\right]$. The fuzzy solution of problem (11) is the family $\widehat{\varphi}_{t}$ given by

$$
\left[\widehat{\varphi}_{t}\left(\mathbf{x}_{0}\right)\right]^{\alpha}=\varphi_{t}\left(\left[\mathbf{x}_{0}\right]^{\alpha}\right)=\left[\frac{\mathbf{x}_{0 \alpha}^{-}}{\mathbf{x}_{0 \alpha}^{-}-\left(\mathbf{x}_{0 \alpha}^{-}-1\right) e^{-\delta t}}, \frac{\mathbf{x}_{0 \alpha}^{+}}{\mathbf{x}_{0 \alpha}^{+}-\left(\mathbf{x}_{0 \alpha}^{+}-1\right) e^{-\delta t}}\right] .
$$

According to Theorem 2, $\widehat{\varphi}_{t}$ is a fuzzy dynamical system.

We will define now an equilibrium point for the fuzzy initial value problem (7) through the extended flow.

Definition 2 (see [12]). We say that $\bar{x} \in \mathscr{F}\left(\mathbb{R}^{n}\right)$ is a fuzzy equilibrium point for $\widehat{\varphi}_{t}$ when $\widehat{\varphi}_{t}(\bar{x})=\bar{x}$, for every $t \geq 0$.

Definition 3 Let $\bar{x} \in \mathscr{F}\left(\mathbb{R}^{n}\right)$ be an equilibrium point of $\widehat{\varphi}_{t}$ :

(1) $\bar{x}$ is said to be Lyapunov stable, if and only if for every $\varepsilon>0$, there exists $\eta>0$ such that, if $D\left(\mathbf{x}_{0}, \bar{x}\right)<\eta$, then for every $t \geq 0$, we have $D\left(\widehat{\varphi}_{t}\left(\mathbf{x}_{0}\right), \bar{x}\right)<\varepsilon$

(2) $\bar{x}$ is said to be asymptotically stable if it is Lyapunov stable and there exists $r>0$ such that, if $D\left(\mathbf{x}_{0}, \bar{x}\right)<r$, then $\lim _{t \longrightarrow+\infty} D\left(\widehat{\varphi}_{t}\left(\mathbf{x}_{0}\right), \bar{x}\right)=0$

(3) $\bar{x}$ is said to be exponentially stable if it is asymptotically stable and there exist $\beta>0, \gamma>0$, and $\sigma>0$ 
such that, if $D\left(\mathbf{x}_{0}, \bar{x}\right)<\beta$, then $D\left(\widehat{\varphi}_{t}\left(\mathbf{x}_{0}\right), \bar{x}\right) \leq$ $\gamma D\left(\mathbf{x}_{0}, \bar{x}\right) e^{-\sigma t}, \forall t \geq 0$

For more details on stability, we refer to [11, 12, 21-25].

Stability of equilibrium points for $\widehat{\varphi}_{t}$ in $\mathscr{F}\left(\mathbb{R}^{n}\right)$ is characterized by the following result.

Theorem 3 (see [10]). Let $\bar{x} \in \mathbb{R}^{n}$ be an equilibrium point of $\varphi_{t}$. So, the following statements are satisfied:

$\bar{x}$ is stable for $\varphi_{t}$ if and only if $\chi_{\{\bar{x}\}}$ is stable for $\widehat{\varphi}_{t}$ $\bar{x}$ is asymptotically stable for $\varphi_{t}$ if and only if $\chi_{\{\bar{x}\}}$ is asymptotically stable for $\widehat{\varphi}_{t}$

Remark 1 (see [10]). Let $U$ be a neighborhood of $\bar{x}$, then $\mathbf{U}=\left\{\mathbf{x} \in \mathscr{F}\left(\mathbb{R}^{n}\right):[\mathbf{x}]^{0} \subseteq U\right\}$ is a neighborhood of $\chi_{\{\bar{x}\}}$.

\section{Main Results}

Before establishing the stability results via Lyapunov functions, we introduce the notion of the fuzzy Lyapunov function, inspired by the definition of Lyapunov functions in the classical case and the relation between the stability of the equilibrium points of the problem (7) and that of the problem (8) given in Theorem 3.

Let $g: \mathbb{R}^{n} \longrightarrow \mathbb{R}$ be a function, and let g: $\mathscr{F}\left(\mathbb{R}^{n}\right) \longrightarrow \mathscr{F}(\mathbb{R})$ be the Zadeh's extension of $f$. We denote by $\hat{g}(\mathbf{x})>0, \forall \mathbf{x} \in \mathscr{F}\left(\mathbb{R}^{n}\right)$ (with 0 being the null element of $\mathbb{R}$ which is also an element of $\mathscr{F}(\mathbb{R})$ with the membership function $\chi_{\{0\}}$ if $\left.g(x)>0, \forall x \in \mathbb{R}^{n}\right)$.

Definition 4. A function V: $\mathscr{F}\left(\mathbb{R}^{n}\right) \longrightarrow \mathscr{F}(\mathbb{R})$ is a fuzzy Lyapunov-candidate function, if $\mathbf{V}$ is Zadeh's extension of a deterministic function $V: \mathbb{R}^{n} \longrightarrow \mathbb{R}$ such that:

(1) $V(0)=0$

(2) $\forall x \in U \backslash\{0\}, V(x)>0$ for a neighborhood $U$ of the origin

$V$ is called the Lyapunov-candidate function associated with $\mathbf{V}$.

Remark 2. Let $\mathbf{V}$ be a fuzzy Lyapunov-candidate function and $V$ be the Lyapunov-candidate function associated. Then,

$$
V(0)=0 \text { if and only if } \mathbf{V}\left(\chi_{\{0\}}\right)=\chi_{\{0\}} \text {. }
$$

Indeed,

$$
\begin{aligned}
\mathbf{V}\left(\chi_{\{0\}}\right)=\chi_{\{0\}} & \Leftrightarrow\left[\mathbf{V}\left(\chi_{\{0\}}\right)\right]^{\alpha}=\left[\chi_{\{0\}}\right]^{\alpha}, \quad \forall \alpha \in[0,1], \\
& \Leftrightarrow V\left(\left[\chi_{\{0\}}\right]^{\alpha}\right)=\left[\chi_{\{0\}}\right]^{\alpha}, \quad \forall \alpha \in[0,1], \\
& \Leftrightarrow V(0)=0 .
\end{aligned}
$$

From Remark 1, Remark 2, and the previous definition, a fuzzy Lyapunov-candidate function V: $\mathscr{F}\left(\mathbb{R}^{n}\right) \longrightarrow \mathscr{F}(\mathbb{R})$ satisfies the following properties:

(1) $\mathbf{V}\left(\chi_{\{0\}}\right)=\chi_{\{0\}}$

(2) $\forall \mathbf{x} \in \mathbf{U} \backslash\left\{\chi_{\{0\}}\right\}, \mathbf{V}(x)>0$ for a neighborhood $\mathrm{U}$ of the origin $\chi_{\{0\}}$
Definition 5. If a fuzzy Lyapunov-candidate function $\mathbf{V}$ satisfies

$$
\dot{V}(x) \leq 0, \quad \forall x \in W \backslash\{0\},
$$

for a neighborhood $W$ of the origin, where $V$ is the Lyapunov-candidate function associated with $\mathbf{V}$.

We say that $\mathbf{V}$ is a fuzzy Lyapunov function, and in this case, $V$ is called the Lyapunov function associated.

\section{Theorem 4}

(1) There exists a fuzzy Lyapunov function for the fuzzy dynamical system $\hat{\varphi}_{t}$ associated with the problem (7), if and only if $\chi_{\{0\}}$ is Lyapunov stable.

(2) $\chi_{\{0\}}$ is asymptotically stable if and only if there is a fuzzy Lyapunov function $\mathbf{V}$ verifying

$$
\dot{V}(x)<0, \quad \forall x \in W \backslash\{0\},
$$

for a neighborhood $W$ of the origin, where $V$ is the Lyapunov function associated with $\mathbf{V}$.

Proof

(1) It is known that there exists a fuzzy Lyapunov function $\mathbf{V}$ if and only if there is a Lyapunov function $V$ associated. In the fact $V$ is a Lyapunov function associated with system (8). Then, 0 is stable for $\varphi_{t}$, and according to Theorem 3, $\chi_{\{0\}}$ is stable for $\widehat{\varphi}_{t}$.

(2) According to Theorem $3, \chi_{\{0\}}$ is asymptotically stable for $\widehat{\varphi}_{t}$ if and only if 0 is asymptotically stable for $\varphi_{t}$. And, this last point is equivalent to say that there is a Lyapunov function $V$ that verifies

$$
\dot{V}(x)<0, \quad \forall x \in W \backslash\{0\},
$$

for a neighborhood $W$ of the origin.

So, let $\mathbf{V}$ be the function constructed by Zadeh's extension applied to $V$, the desired fuzzy Lyapunov function.

Still using the notion of fuzzy Lyapunov functions, we have the following result concerning the exponential stability.

Theorem 5. Let $x_{0} \in \mathbb{R}^{n}$ and $\mathbf{x}_{0} \in \mathscr{F}\left(\mathbb{R}^{n}\right)$ such that $x_{0} \in\left[\mathbf{x}_{0}\right]^{1}$. Then, 0 is exponentially stable for $\varphi_{t}$ if and only if $\chi_{\{0\}}$ is exponentially stable for $\widehat{\varphi}_{t}$.

Proof. It should be noted that the condition $x_{0} \in\left[\mathbf{x}_{0}\right]^{1}$ implies that $x_{0} \in\left[\mathbf{x}_{0}\right]^{\alpha}$ for all $\alpha \in[0,1]$.

$(\Rightarrow)$ Suppose that 0 is exponentially stable for $\varphi_{t}$, then 0 is asymptotically stable for $\varphi_{t}$ and there exist $\beta>0, \gamma>0$, and $\sigma>0$ such that, if $\left\|x_{0}\right\|<\beta$, then

$$
\left\|\varphi_{t}\left(x_{0}\right)\right\|<\gamma\left\|x_{0}\right\| e^{-\sigma t}, \quad \forall t \geq 0 .
$$

If we have

$$
D\left(\mathbf{x}_{0}, \chi_{\{0\}}\right)<\beta,
$$

which means that 


$$
\max \left\{\rho\left(\left[\mathbf{x}_{0}\right]^{\alpha}, \chi_{\{0\}}\right), \rho\left(\chi_{\{0\}},\left[\mathbf{x}_{0}\right]^{\alpha}\right)\right\}<\beta, \quad \forall \alpha \in[0,1] .
$$

So, we get

$$
\sup _{y_{0} \in\left[\mathbf{x}_{0}\right]^{\alpha}}\left\|y_{0}\right\|<\beta, \quad \forall \alpha \in[0,1],
$$

which implies that

$$
\begin{aligned}
\forall \alpha & \in[0,1], \\
\forall y_{0} & \in\left[\mathbf{x}_{0}\right]^{\alpha}, \\
\left\|y_{0}\right\| & <\beta,
\end{aligned}
$$

which leads us to obtain

$$
\begin{array}{r}
\forall \alpha \in[0,1], \\
\forall y_{0} \in\left[\mathbf{x}_{0}\right]^{\alpha}, \\
\left\|\varphi_{t}\left(y_{0}\right)\right\| \leq \gamma\left\|y_{0}\right\| e^{-\sigma t} .
\end{array}
$$

Therefore,

$$
\sup _{z_{0} \in\left[\mathbf{x}_{0}\right]^{\alpha}}\left\|\varphi_{t}\left(z_{0}\right)\right\| \leq \gamma\left\|y_{0}\right\| e^{-\sigma t}, \quad \forall y_{0} \in\left[\mathbf{x}_{0}\right]^{\alpha} .
$$

So, we have

$$
\rho\left(\varphi_{t}\left(\left[\mathbf{x}_{0}\right]^{\alpha}\right),\left[\chi_{\{0\}}\right]^{\alpha}\right) \leq \gamma\left\|y_{0}\right\| e^{-\sigma t}, \quad \forall y_{0} \in\left[\mathbf{x}_{0}\right]^{\alpha} .
$$

Thus, we obtain

$$
\begin{aligned}
& \rho\left(\left[\widehat{\varphi}_{t}\left(\mathbf{x}_{0}\right)\right]^{\alpha},\left[\chi_{\{0\}}\right]^{\alpha}\right) \\
& \leq \gamma\left\|y_{0}\right\| e^{-\sigma t}, \quad \forall y_{0} \in\left[\mathbf{x}_{0}\right]^{\alpha} \\
& \leq \gamma \sup _{y_{0} \in\left[\mathbf{x}_{0}\right]^{\alpha}}\left\|y_{0}\right\| e^{-\sigma t} \\
& \leq \gamma \rho\left(\left[\mathbf{x}_{0}\right]^{\alpha},\left[\chi_{\{0\}}\right]^{\alpha}\right) e^{-\sigma t} \\
& \leq \gamma \max \left\{\rho\left(\left[\mathbf{x}_{0}\right]^{\alpha},\left[\chi_{\{0\}}\right]^{\alpha}\right), \rho\left(\left[\chi_{\{0\}}\right]^{\alpha},\left[\mathbf{x}_{0}\right]^{\alpha}\right)\right\} e^{-\sigma t} .
\end{aligned}
$$

Therefore, for all $\alpha \in[0,1]$,

$$
\rho\left(\left[\widehat{\varphi}_{t}\left(\mathbf{x}_{0}\right)\right]^{\alpha},\left[\chi_{\{0\}}\right]^{\alpha}\right) \leq \gamma D\left(\mathbf{x}_{0}, \chi_{\{0\}}\right) e^{-\sigma t} .
$$

In the same way, for $\alpha \in[0,1]$, we have

$$
\begin{aligned}
\rho\left(\left[\chi_{\{0\}}\right]^{\alpha},\left[\widehat{\varphi}_{t}\left(\mathbf{x}_{0}\right)\right]^{\alpha}\right) & =\inf _{y_{0} \in\left[\mathbf{x}_{0}\right]^{\alpha}}\left\|\varphi_{t}\left(y_{0}\right)\right\| \\
& \leq\left\|\varphi_{t}\left(x_{0}\right)\right\| \\
& \leq \gamma\left\|x_{0}\right\| e^{-\sigma t} \\
& \leq \gamma \sup _{y_{0} \in\left[\mathbf{x}_{0}\right]^{\alpha}}\left\|y_{0}\right\| e^{-\sigma t} .
\end{aligned}
$$

Then,

$$
\rho\left(\left[\chi_{\{0\}}\right]^{\alpha},\left[\widehat{\varphi}_{t}\left(\mathbf{x}_{0}\right)\right]^{\alpha}\right) \leq \gamma D\left(\mathbf{x}_{0}, \chi_{\{0\}}\right) e^{-\sigma t} .
$$

From (28) and (30), we can conclude that

$$
d\left(\left[\widehat{\varphi}_{t}\left(\mathbf{x}_{0}\right)\right]^{\alpha},\left[\chi_{\{0\}}\right]^{\alpha}\right) \leq \gamma D\left(\mathbf{x}_{0}, \chi_{\{0\}}\right) e^{-\sigma t},
$$

and therefore

$$
D\left(\widehat{\varphi}_{t}\left(\mathbf{x}_{0}\right), \chi_{\{0\}}\right) \leq \gamma D\left(\mathbf{x}_{0}, \chi_{\{0\}}\right) e^{-\sigma t},
$$

which shows that $\chi_{\{0\}}$ is exponentially stable for $\widehat{\varphi}_{t}$.

$(\Leftarrow)$ If $\chi_{\{0\}}$ is exponentially stable, then $\chi_{\{0\}}$ is asymptotically stable and there exist $\beta>0, \gamma>0$, and $\sigma>0$ such that, if $D\left(\mathbf{x}_{0}, \chi_{\{0\}}\right)<\beta$, then, for all $t \geq 0$,

$$
D\left(\widehat{\varphi}_{t}\left(\mathbf{x}_{0}\right), \chi_{\{0\}}\right)<\gamma D\left(\mathbf{x}_{0}, \chi_{\{0\}}\right) e^{-\sigma t} .
$$

If we have $\left\|x_{0}\right\|<\beta$, which means that

$$
D\left(\chi_{\left\{x_{0}\right\}}, \chi_{\{0\}}\right)<\beta \text {. }
$$

So, by using (33) and the fact that $x_{0} \in\left[\chi_{\left\{x_{0}\right\}}\right]^{\alpha}$ for all $\alpha \in[0,1]$, we get

$$
D\left(\widehat{\varphi}_{t}\left(\chi_{\left\{x_{0}\right\}}\right), \chi_{\{0\}}\right) \leq \gamma D\left(\chi_{\left\{x_{0}\right\}}, \chi_{\{0\}}\right) e^{-\sigma t} .
$$

Therefore,

$$
\begin{array}{r}
\sup _{0 \leq \alpha \leq 1} d\left(\varphi_{t}\left(\left[\chi_{\left\{x_{0}\right\}}\right]^{\alpha}\right),\left[\chi_{\{0\}}\right]^{\alpha}\right) \\
\leq \gamma \sup _{0 \leq \alpha \leq 1} d\left(\left[\chi_{\left\{x_{0}\right\}}\right]^{\alpha},\left[\chi_{\{0\}}\right]^{\alpha}\right) e^{-\sigma t} .
\end{array}
$$

Consequently,

$$
\left\|\varphi_{t}\left(x_{0}\right)\right\|<\gamma\left\|x_{0}\right\| e^{-\sigma t}, \quad \forall t \geq 0,
$$

which proves the second part.

As a consequence of the previous results, we have the following corollary.

Corollary 1. If 0 is an equilibrium point for $\varphi_{t}$ and $f: \mathbb{R}^{n} \longrightarrow \mathbb{R}^{n}$ with $f^{\prime}(0)=0$, and $\Re\left(\lambda_{i}\right)$ is the real part of the eigenvalue $\lambda_{i}$ associated with 0 :

(1) If $\mathfrak{R}\left(\lambda_{i}\right)<0, \forall i$, then there exists a fuzzy Lyapunov function $\mathbf{V}$ verifying

$$
\dot{V}(x)<0, \quad \forall x \in W \backslash\{0\},
$$

for a neighborhood $W$ of the origin, where $V$ is the Lyapunov function associated with $\mathbf{V}$.

In other words, $\chi_{\{0\}}$ is asymptotically stable for (7).

(2) If $\mathfrak{R}\left(\lambda_{i}\right)>0$, for some $i$, then there is no fuzzy Lyapunov function. In other words, $\chi_{\{0\}}$ is unstable for (7).

To illustrate the elaborate results, we take as application the real model which describes a population, and it is the Malthusian model.

Example 2. We consider the deterministic Malthusian model with a negative variation rate (population in retraction):

$$
\left\{\begin{array}{l}
x^{\prime}(t)=-\lambda x(t), \quad \lambda>0, \\
x(0)=x_{0} \in \mathbb{R} .
\end{array}\right.
$$

The deterministic flow is given by 


$$
\varphi_{t}\left(x_{0}\right)=x_{0} e^{-\lambda t}
$$

Note that 0 is an equilibrium point for $\varphi_{t}$, which is exponentially stable indeed.

Let $V(x)=x^{2}$ on $\mathbb{R}$.

$V$ is a Lyapunov function, and we have $\dot{V}(x)=V^{\prime}(x) \cdot f(x)=-2 \lambda x^{2}<0$, for $x \neq 0$.

So, 0 is asymptotically stable for $\varphi_{t}$. Moreover, we have

$$
\left\|\varphi_{t}\left(x_{0}\right)\right\|=\left\|x_{0} e^{-\lambda t}\right\| \leq\left\|x_{0}\right\| e^{-\lambda t},
$$

which shows that 0 is exponentially stable for $\varphi_{t}$.

But, when we do statistics, we focus on simple and then we generalize the property studied on the entire population, so it is more realistic to consider the initial condition as a fuzzy quantity. And, in this case, the model is of the following form:

$$
\left\{\begin{array}{l}
x^{\prime}(t)=-\lambda x(t), \quad \lambda \in \mathscr{F}\left(\mathbb{R}^{+}\right), \\
x(0)=x_{0} \in \mathscr{F}(\mathbb{R}) .
\end{array}\right.
$$

The fuzzy flow associated with problem (42) is given by

$$
\widehat{\varphi}_{t}\left(\mathbf{x}_{0}\right)=\mathbf{x}_{0} e^{-\lambda t}, \quad \mathbf{x}_{0} \in \mathscr{F}(\mathbb{R}),
$$

where $\left(\widehat{\varphi}_{t}\right)_{t \geq 0}$ is a fuzzy dynamical system.

Let $\mathbf{V}(\mathbf{x})=\widehat{V}(\mathbf{x})$ be the fuzzy Lyapunov function associated with problem (42) given by

$$
[\mathbf{V}(\mathbf{x})]^{\alpha}=V\left([\mathbf{x}]^{\alpha}\right)=\left[\min P_{\alpha}, \max P_{\alpha}\right],
$$

for $[\mathbf{x}]^{\alpha}=\left[\mathbf{x}_{-}^{\alpha}, \mathbf{x}_{+}^{\alpha}\right], \alpha \in[0,1]$, where

$$
P_{\alpha}=\left\{\left(\mathbf{x}_{-}^{\alpha}\right)^{2},\left(\mathbf{x}_{+}^{\alpha}\right)^{2}, \mathbf{x}_{-}^{\alpha} \cdot \mathbf{x}_{+}^{\alpha}\right\} .
$$

Note that $\chi_{\{0\}}$ is an equilibrium point for $\widehat{\varphi}_{t}$.

By using Theorem 4, we conclude that $\chi_{\{0\}}$ is asymptotically stable for $\widehat{\varphi}_{t}$, and we have

$$
\begin{aligned}
D\left(\widehat{\varphi}_{t}\left(\mathbf{x}_{0}\right), \chi_{\{0\}}\right) & =D\left(\mathbf{x}_{0} e^{-\lambda t}, \chi_{\{0\}}\right) \\
& =\sup _{\alpha \in[0,1]} d\left(e^{-\lambda t}\left[\mathbf{x}_{0}\right]^{\alpha}, e^{-\lambda t}\{0\}\right) \\
& \leq \sup _{\alpha \in[0,1]} d\left(\left[\mathbf{x}_{0}\right]^{\alpha},\{0\}\right) e^{-\lambda t} \\
& =D\left(\mathbf{x}_{0}, \chi_{\{0\}}\right) e^{-\lambda t} .
\end{aligned}
$$

That is to say, $\chi_{\{0\}}$ is exponentially stable for $\widehat{\varphi}_{t}$.

Note that we can deduce directly from Theorem 5 the last point.

Figure 1 represents the dynamic of $\widehat{\varphi}_{t}$ around $\chi_{\{0\}}$, where we considered in problem (42) the following parameters: $x_{0}$ is "around 35," which can be modeled by a triangular fuzzy number $x_{0}=(30 ; 35 ; 40)$, whose $\alpha$-cuts are given by

$$
\left[x_{0}\right]^{\alpha}=\left[x_{0 \alpha}^{-}, x_{0 \alpha}^{+}\right]=[30+5 \alpha, 40-5 \alpha], \quad \text { for } \alpha \in[0,1] \text {. }
$$

And, $\lambda$ is the symmetric triangular fuzzy number defined by $\lambda=(0.4 ; 0.5 ; 0.6)$, whose $\alpha$-cuts are given by

$$
[\lambda]^{\alpha}=[0.4+0.1 \alpha, 0.6-0.1 \alpha], \quad \text { for } \alpha \in[0,1] .
$$

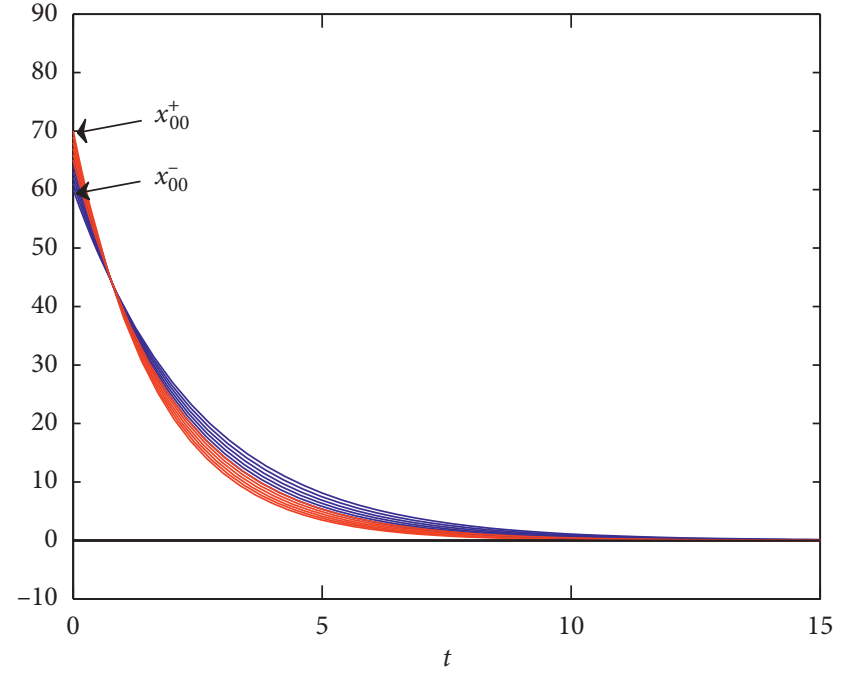

Figure 1: Dynamic of $\hat{\varphi}_{t}$ around $\chi_{\{0\}}$.

Example 3. Consider the following system:

$$
\left\{\begin{array}{l}
x^{\prime}(t)=-x+y, \\
y^{\prime}(t)=-x-y, \\
x(0)=x_{0}, y(0)=y_{0} .
\end{array}\right.
$$

This system can be written as

$$
\left\{\begin{array}{l}
X^{\prime}(t)=G(X(t)), \\
X(0)=X_{0}=\left(x_{0}, y_{0}\right) \in \mathbb{R}^{2},
\end{array}\right.
$$

where $X(t)=(x(t), y(t)) \in \mathbb{R}^{2}$ and

$$
G(X(t))=(-x(t)+y(t),-x(t)-y(t)), \quad \text { for } t \in \mathbb{R}^{+} .
$$

We consider the fuzzy initial value problem:

$$
\left\{\begin{array}{l}
\mathbf{X}^{\prime}=\widehat{G}(\mathbf{X}(t)), \\
\mathbf{X}(0)=\mathbf{X}_{0} \in \mathscr{F}\left(\mathbb{R}^{2}\right),
\end{array}\right.
$$

where $\widehat{G}$ is Zadeh's extension applied to $G$.

We study the stability of the equilibrium point $(0,0)$ for system (50).

We define on $\mathbb{R}^{2}$ the following function:

$$
V(X)=V(x, y)=x^{2}+y^{2} .
$$

It is easy to verify that $V$ is a Lyapunov function, and we have

$$
\dot{V}(x, y)=2 x(-x+y)+2 y(-x-y)<0, \quad \text { for } x \neq 0, y \neq 0 .
$$

And, $(0,0)$ is therefore asymptotically stable.

Now, we want to study the stability of system (51).

We have $\mathbf{X}_{0} \in \mathscr{F}\left(\mathbb{R}^{2}\right)$, then $\chi_{\{(0,0)\}}$ will be an equilibrium point of the fuzzy dynamical system associated with (51).

Let $\mathbf{V}(\mathbf{X})=\widehat{V}(\mathbf{X})$, for $\mathbf{X} \in \mathscr{F}\left(\mathbb{R}^{2}\right)$. 
V is the fuzzy Lyapunov function associated with (51), whose $\alpha$-cuts are

$$
[\mathbf{V}(\mathbf{X})]^{\alpha}=V\left([\mathbf{X}]^{\alpha}\right), \quad \text { for } \alpha \in[0,1] .
$$

From Theorem 4, we deduce that $\chi_{\{(0,0)\}}$ is asymptotically stable for problem (52).

We can also show the asymptotic stability of $\chi_{\{(0,0)\}}$ using Corollary 1 indeed.

It is easy to check that $\lambda_{1}=-1+i$ and $\lambda_{2}=-1-i$ are the eigenvalues associated with system (50).

We have $\mathfrak{R}\left(\lambda_{1}\right)=\mathfrak{R}\left(\lambda_{2}\right)<0$, and therefore $\chi_{\{(0,0)\}}$ is asymptotically stable for the fuzzy initial value problem (52).

Now, we show that $\chi_{\{(0,0)\}}$ is exponentially stable for system (51) indeed.

We have

$$
\dot{V}(X)=\dot{V}(x, y)=-2\left(x^{2}+y^{2}\right) \leq-2 V(X) .
$$

By using Grönwall's inequality, for $X_{0}=\left(x_{0}, y_{0}\right) \in \mathbb{R}^{2}$, we can obtain

$$
V(X) \leq V\left(X_{0}\right) e^{-2 t}, \quad \text { for } t \geq 0,
$$

which leads us to have

$$
\|X\|=\|(x, y)\| \leq\left\|\left(x_{0}, y_{0}\right)\right\| e^{-t}=\left\|X_{0}\right\| e^{-t}, \quad \text { for } t \geq 0 \text {. }
$$

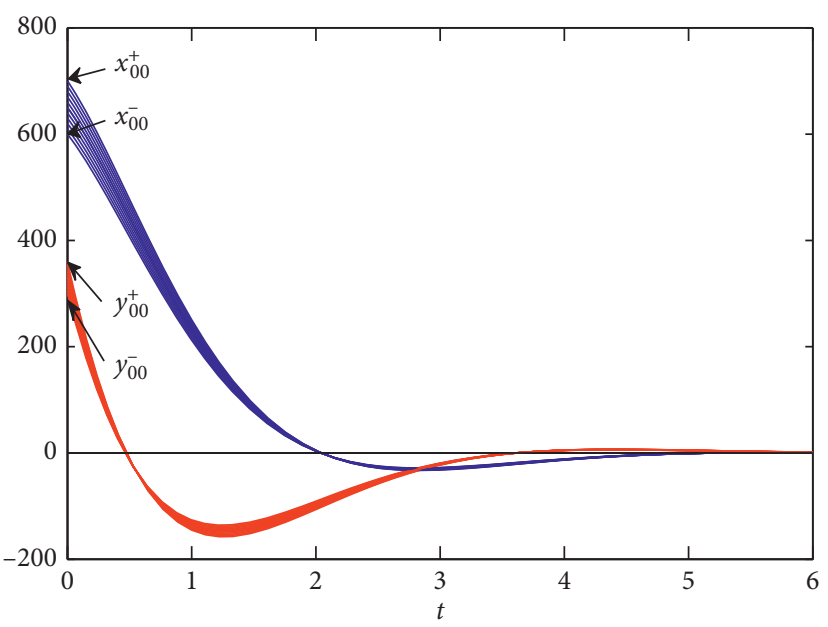

Figure 2: Dynamic of $\widehat{\varphi}_{t}$ around $\chi_{\{(0,0)\}}$.

Then, $(0,0)$ is exponentially stable for $(50)$.

Using Theorem 5, we can conclude that $\chi_{\{(0,0)\}}$ is exponentially stable for (51).

Let $\quad \mathbf{X}_{0} \in \mathscr{F}\left(\mathbb{R}^{2}\right)$, whose membership function $\mu: \mathbb{R}^{2} \longrightarrow[0,1]$ is defined as, for $(x, y) \in \mathbb{R}^{2}$,

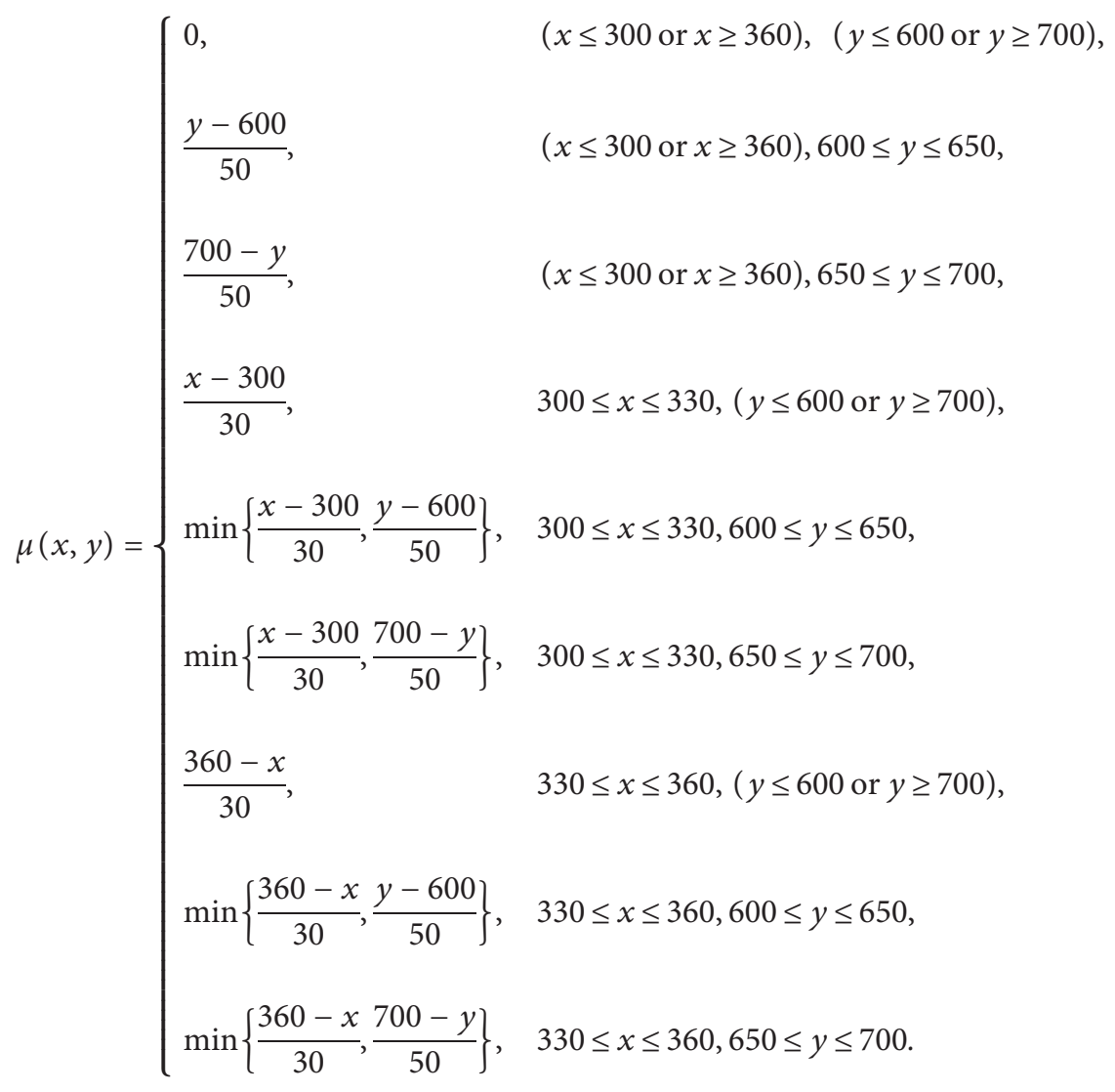


It is easy to verify that, for all $\alpha \in[0,1]$,

$$
\left[\mathbf{X}_{0}\right]^{\alpha}=[300+30 \alpha, 360-30 \alpha] \times[600+50 \alpha, 700-50 \alpha] \text {. }
$$

Figure 2 shows the stability of $\chi_{\{(0,0)\}}$ for problem (52).

\section{Conclusion}

In this work, we studied the stability of fuzzy dynamical systems using Lyapunov functions. We began by defining the fuzzy Lyapunov function in a way analogous to that of the classical case. We achieved to show some equivalence results between stability by different types whether it is stability, asymptotic stability, or exponential stability and the existence of a fuzzy Lyapunov function. Our results will be used in further works to generalize the notion of the stability of fuzzy dynamical systems.

\section{Data Availability}

The data used to support the findings of this study are available from the corresponding author upon request.

\section{Conflicts of Interest}

The authors declare that they have no conflicts of interest.

\section{Authors' Contributions}

All authors contributed equally to the writing of this paper. All authors read and approved the final manuscript.

\section{References}

[1] P. E. Kloeden, "Fuzzy dynamical systems," Fuzzy Sets and Systems, vol. 7, no. 3, pp. 275-296, 1982.

[2] De Barros, C. Laecio, C. R. Bassanezi, and A. P. Tonelli, "Continuity of the Zadeh's extension," in Proceedings of the IFSA 1997 Congress, p. 6, Prague, Czech Republic, June 1997.

[3] A. El Allaoui, S. Melliani, and L. S. Chadli, "Complex fuzzy dynamical systems and Stability of the equilibrium Point," Journal of Fuzzy Set Valued Analysis, vol. 3, pp. 223-233, 2016.

[4] S. Melliani, A. El Allaoui, and L. S. Chadii, "Relation between fuzzy semigroups and fuzzy dynamical systems," Nonlinear Dynamics and Systems Theory, vol. 17, no. 1, pp. 60-69, 2017.

[5] H. T. Nguyen, "A note on the extension principle for fuzzy sets," Journal of Mathematical Analysis and Applications, vol. 2, no. 64, pp. 369-380, 1978.

[6] Román-Flores, Heriberto, Barros, C. Laécio, and R. C. Bassanezi, “A note on Zadeh's extensions," Fuzzy Sets and Systems, vol. 3, no. 117, pp. 327-331, 2001.

[7] S. Song, C. Wu, and E. S. Lee, "Asymptotic equilibrium and stability of fuzzy differential equations," Computers \& Mathematics with Applications, vol. 7, no. 49, pp. 1267-1277, 2005.

[8] A. El Allaoui, S. Melliani, and L. S. Chadli, "Fuzzy dynamical systems and Invariant attractor sets for fuzzy strongly continuous semigroups," Journal of Fuzzy Set Valued Analysis, vol. 2016, no. 2, pp. 148-155, 2016.

[9] E. Hüllermeier, "An approach to modelling and simulation of uncertain dynamical systems," International Journal of
Uncertainty, Fuzziness and Knowledge-Based Systems, vol. 5, no. 2, pp. 117-137, 1997.

[10] M. S. Cecconello, J. Leite, R. C. Bassanezi, and A. J. V. Brandão, "Invariant and attractor sets for fuzzy dynamical systems," Fuzzy Sets and Systems, vol. 265, pp. 99109, 2015

[11] De Barros, L. Carvalho, R. Bassanezi, and W. Alexander, "A first course in fuzzy logic, fuzzy dynamical systems, and biomathematics," Studies in Fuzziness and Soft Computing, Springer, vol. 347, Berlin, Heidelberg, 2017.

[12] M. S. Cecconello, R. C. Bassanezi, A. J. V. Brandão, and J. Leite, "On the stability of fuzzy dynamical systems," Fuzzy Sets and Systems, vol. 248, pp. 106-121, 2014.

[13] M. S. Cecconello, R. C. Bassanezi, A. V. Brandão, and J. Leite, "Periodic orbits for fuzzy flows," Fuzzy Sets and Systems, vol. 230, pp. 21-38, 2013.

[14] P. Diamond, "Stability and periodicity in fuzzy differential equations," IEEE Transactions on Fuzzy Systems, vol. 5, no. 8, pp. 583-590, 2000.

[15] M. Mizukoshi, L.C. Carvalho, Bassanezi, and R. Carlos, "Stability of fuzzy dynamic systems," International Journal of Uncertainty, Fuzziness and Knowledge-Based Systems, vol. 1, no. 17, pp. 69-83, 2009.

[16] M. L. Puri and D. A. Ralescu, "Fuzzy random variables," Journal of Mathematical Analysis and Applications, vol. 114, no. 2, pp. 409-422, 1986.

[17] J. J. Buckley and T. Feuring, "Fuzzy differential equations," Fuzzy Sets and Systems, vol. 1, no. 110, pp. 43-54, 2000.

[18] M. S. Cecconello, J. Leite, R. C. Bassanezi, and J. de D. M. Silva, "About projections of solutions for fuzzy differential equations," Journal of Applied Mathematics, vol. 2013, Article ID 184950, 9 pages, 2013.

[19] O. Kaleva, "The cauchy problem for fuzzy differential equations," Fuzzy Sets and Systems, vol. 3, no. 35, pp. 389-396, 1990.

[20] O. Kaleva, "Fuzzy differential equations," Fuzzy Sets and Systems, vol. 24, no. 3, pp. 301-317, 1987.

[21] O. Kaleva, "A note on fuzzy differential equations," Nonlinear Analysis: Theory, Methods\\&Applications, vol. 5, no. 64, pp. 895-900, 2006.

[22] H. Aminikhah, A. Refahi Sheikhani, and H. Rezazadeh, "Stability analysis of linear distributed order system with multiple time delays," UPB Scientific Bulletin, Series A, vol. 77, no. 2, 2015

[23] H. Aminikhah, A. Refahi Sheikhani, and H. Rezazadeh, "Stability analysis of distributed order fractional chen system," The Scientific World Journal, vol. 2013, p. 13, 2013.

[24] V. Mohammadnezhad, M. Eslami, and H. Rezazadeh, "Stability analysis of linear conformable fractional differential equations system with time delays," Boletim da Sociedade Paranaense de Matemática, vol. 38, no. 6, pp. 159-171, 2020.

[25] H. Rezazadeh, H. Aminikhah, and A. R. Sheikhani, "Stability analysis of Hilfer fractional differential systems," Mathematical Communications, vol. 21, no. 1, 2016. 